\title{
Aus dem Vorwort zur 4. amerikanischen Auflage
}

Es ist jetzt zwanzig Jahre her, daß der Senior unter den Autoren mit dem Verlag Harper \& Row die erste Auflage von Anorganische Chemie - Prinzipien von Struktur und Reaktivität produziert hat. Seitdem ist der Senior Autor 20 Jahre älter geworden, haben sich zwei neue Autoren dem Projekt angeschlossen, hat der Verlag Harper \& Row seinen Namen in Harper Collins geändert und - bei weitem am wichtigsten - ist die anorganische Chemie weiter gewachsen. Es wird für einen einzelnen Autor zunehmend schwieriger, wenn nicht gar unmöglich, alle Bereiche der anorganischen Chemie zu verfolgen. Die beiden neuen Autoren bringen in das Buch ihre Kenntnisse in Koordinationschemie, in metallorganischer Chemie und in physikalischen Methoden ein, sowie frische Ansichten zu einer Reihe von anderen Themen. Trotzdem wurde die Philiosophie des Buches beibehalten: dem Leser die wichtigsten Aspekte der anorganischen Chemie in einer leicht lesbaren Form zu vermitteln und dabei klarzustellen, da $ß$ die anorganische Chemie ein faszinierendes Forschungsgebiet ist und nicht ein abgeschlossenes Wissensfeld.

Wir drei Autoren bringen völlig verschiedene Studienerfahrungen in dieses Buch ein. Einer von uns erwarb den Bachelor of Science (B.Sc.) an einer Hochschule mit Promotionsrecht, einer an einem privaten College (ohne Promotionsrecht) und einer an einem staatlichen College. Wir haben anorganische Chemie im Grund- und Hauptstudium an verschiedenen Orten unterrichtet. Als wir uns zusammensetzten, um die Revision dieses Buches zu besprechen, waren wir uns in folgenden Punkten einig: (1) Das Buch sollte inhaltlich auf den neuesten Stand gebracht werden. (2) Der Inhalt sollte weiterhin durch Literaturangaben untermauert werden; diese sollten auf der jeweiligen Seite erscheinen, zu der sie gehören und relevante Zitate sollten nicht einfach deswegen entfallen, weil sie bereits in der vorigen Auflage erschienen waren. (3) Neue Abbildungen, oftmals aus der Originalliteratur, sollten aufgenommen werden. (4) Eine größere Anzahl von Aufgaben, viele davon neu, sollten am Ende der Kapitel erscheinen. Die Lösung mancher Aufgaben sollte die Benutzung einer Bibliothek erfordern, während andere die grundlegenden Fragestellungen zu den entsprechenden Themen ansprechen sollten. (5) Ein Kapitel über Symmetrie sollte eingefügt werden. (6) Die Festkörperchemie sollte einen größeren Umfang erhalten. (7) Der Abschnitt zur Kinetik sollte ausgebaut werden. (8) Die Beschreibung der Lanthanoide und Actinoide sollte in die Kapitel der entsprechenden Übergangsmetalle integriert werden.

Ein allgemeiner Konsens sowohl zwischen Autoren als auch Benutzern ist einfacher zu erreichen als eine Einigung auf spezifische Details. Unsere Diskussionen über das Kapitel zur Symmetrie sind dafür ein gutes Beispiel. Wir waren uns einig, daß die Lehre über Symmetrie an den meisten Hochschulen Aufgabe der anorganischen Chemie geworden ist. Aber wie viel sollte gebracht werden und in welchem Ausmaß sollte der Rest des Buches von diesem Symmetriekapitel abhängen? Als Minimum hielten wir eine gute Einführung in das Thema Punktgruppen für unerläßlich. Auch wollten wir einige Anwendungen für Charaktertafeln einbauen, aber wiederum nicht so weit gehen, $\mathrm{da} ß$ die anorganische Chemie in diesem Buch nicht mehr ohne Charaktertafeln unterrichtet werden kann. Diese Anwendungen finden sich verstreut im Text und können gegebenenfalls auch übergangen werden. Das Symmetriekapitel, wie es nun vorliegt, 
konzentriert sich darauf, den Leser mit möglichst vielen Anwendungen der Symmetrie vertraut zu machen, unter anderem in der Spektroskopie und Kristallographie, ohne jedoch den Anspruch zu erheben, eine umfassende Darstellung dieses Themas zu sein.

Studierende, die dieses Buch benutzen, haben vermutlich völlig unterschiedliche Vorkenntnisse. Einige werden bereits Kenntnisse in anorganischer, physikalischer und organischer Chemie besitzen, für andere mag es der erste Kontakt mit der anorganischen Chemie sein. Andere wieder haben vielleicht nur wenig Vorkenntnisse in Bindungstheorie. Aus diesem Grunde bringen die ersten Kapitel die Grundlagen des Atombaues und der Molekülstruktur aus der Perspektive eines Anorganikers. Leser mit entsprechenden Kenntnissen mögen diese Kapitel zur Wiederholung benutzen oder als Mörtel, um die Lücken in ihrem Wissen zu kitten. Die mittleren Kapitel dieses Buches stellen das „Herz der anorganischen Chemie“ dar: die Festkörperchemie jenseits der einfachen Salze, die Säure-Base-Chemie in verschiedenen Lösungsmitteln und in der Gasphase sowie die Koordinationschemie mit ihren verschiedenen Aspekten Bindung, Spektren, Magnetismus, Struktur und Reaktionen.

In Übereinstimmung mit der Philosophie einer themenorientierten Gliederung dieses Buches sind die letzten sechs Kapitel im wesentlichen voneinander unabhängig. Insgesamt sind wir davon ausgegangen, daß es am besten ist, eine große Zahl von Gebieten zu besprechen, aus der jeder seine Lieblingsthemen auswählen kann.

Wir möchten unseren Kollegen an der University of Maryland at College Park (UMCP) und an der Eastern Illinois University (EIU) danken, die uns in vielfältiger Weise geholfen haben. Außerdem danken wir Bryan Eichhorn (UMCP), William Harwood (UMCP), Mark McGuire (EIU), Robert Pilato (UMCP) und Rinaldo Poli (UMCP) für ihre spezielle Hilfe bei der Anfertigung dieser Auflage. Auch möchten wir den Kollegen danken, die wir während unserer Forschungssemester in anderen Departements besucht haben: Fred Hawthorne, Herb Kaesz, Charles Strouse, Joan Selverstone Valentine und Jeff Zink (University of California at Los Angeles), sowie Oren Anderson, Gary Maciel, Jack Norton, Tony Rappé und Steve Straus (Colorado State University). Wir möchten auch den Chemistry Departements von UCLA und CSU, dem Zoology Departement der Southern Illinois University sowie unseren eigenen Departements dafür danken, daß sie die Forschungsaufenthalte an deren Universitäten ermöglicht haben.

Für Ihre Hilfe bei der Übernahme von Abbildungen aus ihren Veröffentlichungen danken wir Michael W. Anderson (University of Cambridge), Anthony Arduengo (E. I. du Pont de Nemours), B. Dubost (Pechiney Institute), Jacek Klinowski (University of Cambridge), John Newsam (BIOSYM Technologies), Joseph J. Pluth (University of Chicago), Arnold L. Rheingold (University of Delaware), P. Saintford (Pechiney Institute), Carlotte L. Stern (University of Illinois, Urbana-Champaign), Sir John Meurig Thomas (The Royal Institution of Great Britain) und Scott Wilson (University of Illinois, Urbana-Champaign).

Die Abfassung dieses Textes hat von den hilfreichen Ratschlägen vieler Begutachter profitiert. Zu ihnen gehören Ivan Bernal, Donals H. Berry, Patricia A. Bianconi, Andrew B. Bocarsly, P. Michael Boorman, Jeremy Burdett, Ben DeGraff, Russel S. Drago, Daniel C. Harris, Roald Hoffmann, Joel F. Liebman, John Milne, Terrance Murphy, Jack Pladziewicz, Philip Power, Arnold L. Rheingold, Richard Thompson, Glen Vogel, Marc Walters, James H. Weber und Jeff Zink.

Schließlich gibt es viele Kollegen und Studenten, die bei der Niederschrift des ersten 
Textes sowie seiner weiteren Vervollkommnung geholfen haben. Sie wissen selbst, wer sie sind, und wir hoffen, daß sie unseren aufrichtigsten Dank für alles das, was sie getan haben, anzunehmen bereit sind.

James E. Huheey

Ellen A. Keiter

Richard L. Keiter 